\title{
Atypical pneumonia due to human bocavirus in an immunocompromised patient
}

\author{
Kaien Gu BSc, Paul Van Caeseele MD, Kerry Dust MSc, Julie Ho MD
}

Cite as: CMAJ 2017 May 15;189:E697-9. doi: 10.1503/cmaj.161134

A 60-year-old woman presented to the emergency department with difficulty breathing and a nonproductive cough that worsened when she lay flat. The symptoms progressed from a previous mild respiratory tract illness despite the patient having received an empiric five-day course of azithromycin that was completed three weeks before her presentation.

The patient was immunocompromised because of an allogeneic bone marrow transplant for aplastic anemia and a deceased-donor renal transplant for end-stage renal disease from post-transplant diabetes; as a result, she was taking mycophenolate mofetil, tacrolimus and prednisone. In addition, she had a history of latent tuberculosis prophylactically treated with isoniazid, chronic inactive hepatitis B treated with lamivudine, hypertension, hypercholesterolemia and gout. She had no history of cardiac disease.

On presentation, the patient's blood pressure was 166/85 mm Hg, her heart rate was 99 beats/min and regular, her temperature was $37^{\circ} \mathrm{C}$ and her oxygen saturation was $87 \%$ on room air. A physical examination showed decreased breath sounds and crackles bilaterally on auscultation. Her white blood cell count was slightly elevated -12.5 (normal 4.5-11.0) $\times 10^{9} / \mathrm{L}$ ) - on presentation and normal thereafter. Chest radiography in the emergency department showed bilateral pulmonary infiltrates that were interpreted as congestive heart failure (Figure 1).

The patient was given diuretics intravenously and supportive care for presumed congestive heart failure, but her condition showed no improvement. Owing to a recent cluster of Pneumocystis jirovecii pneumonia cases in the hospital's renal transplant population, there was a high index of suspicion for this condition, and computed tomography (CT) of the chest was performed the following day. The CT imaging showed diffuse bilateral peribronchial consolidation that was consistent with $P$. jirovecii pneumonia rather than pulmonary edema or hemorrhage. The patient was empirically started on trimethoprim-sulfamethoxazole intravenously, in addition to prednisone orally, for possible $P$. jirovecii pneumonia, and ceftriaxone and azithromycin for possible community-acquired pneumonia. Because it was influenza season, the patient was also started on oseltamivir and isolated with droplet precautions.

Bronchoscopy was performed the following day, which showed purulent secretions that were more prominent on the left. Bronchoalveolar lavage samples were analyzed by polymerase chain reaction (PCR) testing (Allplex Respiratory Full

\section{KEY POINTS}

- Human bocavirus is an uncommon cause of atypical pneumonia in adults.

- A high clinical index of suspicion for atypical infections should be maintained for immuncompromised patients, because they may not present with typical symptoms of infection.

- Rapid use of specific therapies for atypical infections is critical for optimal patient outcomes.

- Starting infection precaution procedures early may limit nosocomial spread, particularly to other immunocompromised patients.

Panel Assay, Seegene, Korea) and showed a positive result for human bocavirus. Cytomegalovirus was also identified in the bronchoalveolar lavage. However, the patient was known to have been postive for the virus before her transplant. No other bacterial, fungal or viral pathogenic coinfections were identified; tests were negative for acid-fast bacilli and Legionella. A five-day course of oseltamivir was already complete by the time PCR results were available. The patient's condition improved with supportive therapy, and she was discharged home 13 days after her initial presentation. Her clinical picture was not consistent with tissue-invasive cytomegalovirus disease, and her condition improved in the absence of specific therapy.

\section{Discussion}

Atypical pneumonias may be characterized by a preceding mild respiratory illness, followed by pneumonia with dyspnea, cough and lack of sputum production. Mycoplasma pneumoniae, Chlamydia pneumoniae, Legionella pneumophila and respiratory viruses are among the most important causes of atypical pneumonias, ${ }^{1}$ but these may vary in an immunocompromised patient.

Noninfluenza viral respiratory tract infections are a leading cause of atypical pneumonia and hospital admission, and have a considerable burden of illness. In the United States alone, it has been estimated that more than 500 million noninfluenza viral respiratory tract infections occur each year, with an economic impact of nearly US\$40 billion. ${ }^{2}$ In addition, up to one-third of recipients of bone marrow transplants admitted to hospital have a viral respira- 
tory tract infection, suggesting a high burden of illness in this vulnerable population. ${ }^{3}$ Common pathogens include respiratory syncytial virus, rhinoviruses, parainfluenza viruses and parvoviruses; however, many infections have no known identifiable agent. ${ }^{4}$

Human bocavirus was first discovered in 2005 and belongs to the Parvoviridae family. ${ }^{5}$ It has been described as an emerging virus, associated with respiratory illness and gastroenteritis in children. ${ }^{6}$ Human bocavirus respiratory infection has been reported in hematopoietic stem cell ${ }^{7}$ and lung ${ }^{8,9}$ transplant recipients, and is an uncommon cause of encephalitis. Human bocavirus infection can present with severe respiratory infection that requires intensive care and mechanical ventilation, with associated morbidity and death.

The prevalence of human bocavirus infection is more common in children, especially those aged three years and younger (2.3\%-5.7\%) compared with adults $(0.4 \%-1.5 \%), 6,10,11$ which suggests that human bocavirus is an uncommon cause of respiratory illness. True prevalence, however, may be underestimated because of underdetection. Human bocavirus respiratory illness can range from upper respiratory tract disease to severe bronchiolitis and pneumonia. ${ }^{11}$ Data from Canadian and American populations suggest that human bocavirus infection may be more likely to occur during the fall and winter months, ${ }^{10,11}$ in association with colder temperatures. ${ }^{12}$

Patients with human bocavirus infection typically present with expiratory wheezing, cough and fever; however, our patient was afebrile, with a single elevated leukocyte count of $12.5 \times 10^{9} / \mathrm{L}$ on presentation; thus, her bilateral pulmonary infiltrates were initially interpreted as congestive heart failure. Our patient's case highlights the importance of maintaining a high clinical index of suspi- cion for atypical infections in immunocompromised patients to facilitate rapid institution of appropriate therapy. Furthermore, early identification of atypical infections may help decrease nosocomial transmission with appropriate isolation precautions.

There has been controversy in the literature regarding human bocavirus as a causal pathogen owing to a high observed coinfection rate and the inability to fulfill classical Koch postulates given the challenges of viral culture..$^{13}$ Indeed, human bocavirus has been identified in nasal swabs and blood from asymptomatic people. ${ }^{14,15}$ Our patient's case shows detection of human bocavirus in the absence of any relevant coinfections, a clear association with respiratory symptoms, hypoxia and an atypical pneumonic infiltrate. Human bocavirus has not been detected as a colonizer or commensal in the bronchoalveolar lavage specimens of immunocompetent adults. ${ }^{9}$ Furthermore, it has not been detected in asymptomatic, immunocompromised lung transplant recipients undergoing surveillance bronchoscopies ${ }^{5}$ or in nasal wash surveillance samples of adults before undergoing hematopoietic stem cell transplantation. ${ }^{7}$ Taken together, these data suggest that human bocavirus may be our patient's pathogenic agent and not simply an epiphenomenon related to respiratory tract colonization by the virus.

There are no current specific therapies for human bocavirus infection. Our patient received empiric oseltamivir because of the coincident timing of her clinical presentation with influenza season, and she completed a five-day course. It is uncertain whether this contributed to the clinical resolution of her symptoms, or if her condition would have improved irrespective of oseltamivir.

Our patient's case highlights the broad differential diagnosis for an atypical pneumonia in an immunocompromised patient
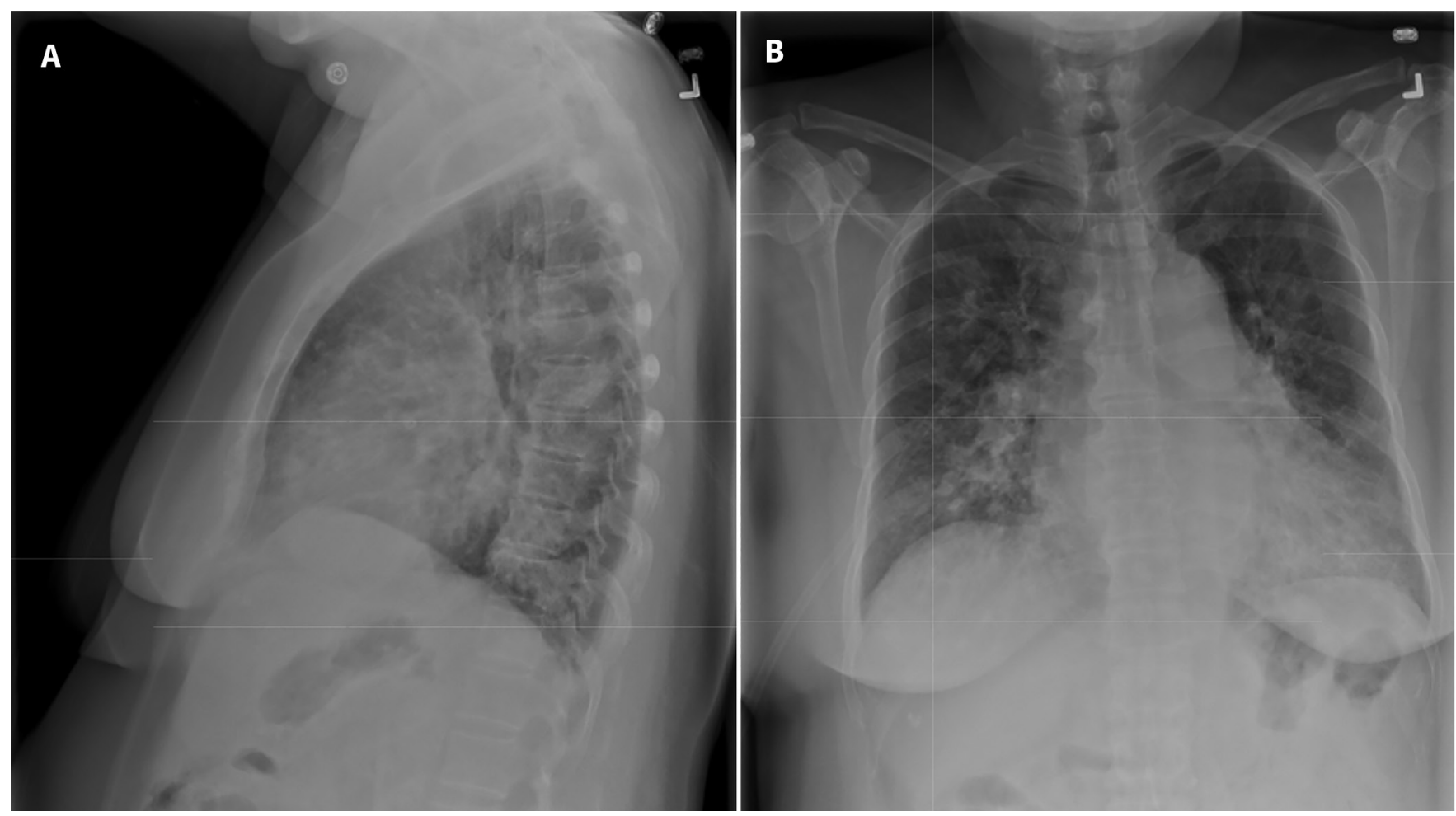

Figure 1: Lateral (A) and posteroanterior (B) radiographs of the chest of a 60-year-old immunocompromised woman who presented to the emergency department with difficulty breathing and a nonproductive cough. Bilateral pulmonary infiltrates that were intially interpreted as congestive heart failure can be seen. The patient was later diagnosed with atypical pneumonia caused by human bocavirus. 
and shows the importance of emerging viral infections. Further clinical-pathologic characterization of emerging viral infections is needed for these vulnerable populations.

\section{References}

1. Musher DM, Thorner AM. Community-acquired pneumonia. N Engl J Med 2014;371: 1619-28.

2. Fendrick AM, Monto AS, Nightengale B, et al. The economic burden of noninfluenza-related viral respiratory tract infection in the United States. Arch Intern Med 2003;163:487-94.

3. Shah DP, Ghantoji SS, Mulanovich VE. Management of respiratory viral infections in hematopoietic cell transplant recipients. Am J Blood Res 2012;2:203-18.

4. Iwane MK, Edwards KM, Szilagi PG, et al. Population-based surveillance for hospitalizations associated with respiratory syncytial virus, influenza virus, and parainfluenza viruses among young children. Pediatrics 2004;113:1758-64.

5. Allander T, Tammi MT, Eriksson M, et al. Cloning of a human parvovirus by molecular screening of respiratory tract samples. Proc Natl Acad Sci U S A 2005;102:12891-6.

6. Principi N, Piralla A, Zampiero A, et al. Bocavirus Infection in Otherwise Healthy Children with Respiratory Disease. PLoS One 2015;10:e0135640.
7. Campbell AP, Guthrie KA, Englund JA, et al. Clinical outcomes associated with respiratory virus detection before allogeneic hematopoietic stem cell transplant. Clin Infect Dis 2015;61:192-202.

8. Vu DL, Bridevaux PO, Aubert JD, et al. Respiratory viruses in lung transplant recipients: a critical review and pooled analysis of clinical studies. Am J Transplant 2011;11:1071-8.

9. Garbino J, Soccal PM, Aubert JD, et al. Respiratory viruses in bronchoalveolar lavage: a hospital-based cohort study in adults. Thorax 2009;64:399-404.

10. Chow BD, Huang YT, Esper FP. Evidence of human bocavirus circulating in children and adults, Cleveland, Ohio. J Clin Virol 2008;43:302-6.

11. Bastien N, Chui N, Robinson JL, et al. Detection of human bocavirus in Canadian children in a 1-year study. J Clin Microbiol 2007;45:610-3.

12. Sundell N, Andersson LM, Brittain-Long R, et al. A four-year seasonal survey of the relationship between outdoor climate and epidemiology of viral respiratory tract infections in a temperate climate. J Clin Virol 2016;84:59-63.

13. Broccolo F, Falcone V, Esposito S, et al. Human bocaviruses: possible etiologic role in respiratory infection. J Clin Virol 2015;72:75-81.

14. Byington $\mathrm{CL}$, Ampofo $\mathrm{K}$, Stockman $\mathrm{C}$, et al. Community surveillance of respiratory viruses among families in the Utah Better Identification of Germs-Longitudinal Viral Epidemiology (BIG-LoVE) Study. Clin Infect Dis 2015;61:1217-24.

15. Bonvicini F, Manaresi E, Gentilomi, GA, et al. Evidence of human bocavirus viremia in healthy blood donors. Diag Microbiol Infect Dis 2011;71:460-2.

\section{Competing interests: None declared.}

This article has been peer reviewed.

The authors have obtained patient consent.

Affiliations: Section of Nephrology, Department of Internal Medicine (Gu, Ho), University of Manitoba; Cadham Provincial Laboratory (Van Caeseele, Dust), Manitoba Health; Departments of Medical Microbiology (Van Caeseele); and Immunology (Ho), University of Manitoba, Winnipeg, Man.
Contributors: Kaien Gu and Julie Ho wrote the report and were involved in the patient's management. Paul Van Caeseele and Kerry Dust contributed to writing the report and providing content expertise on human bocavirus. All of the authors approved the version to be published and agreed to act as guarantors of the work.

Funding: Julie Ho is supported by a Canadian Institutes of Health Research New Investigator Salary Award.

Correspondence to: Julie Ho, jho@hsc.mb.ca 